J3eA, Journal sur l'enseignement des sciences et technologies de l'information et des systèmes, Volume 4, Hors-Série 2, 2 (2005)

DOI : http://dx.doi.org/10.1051/bib-j3ea:2005702

(C) EDP Sciences, 2005

Expérience de développement et de mise en place de modules de formation par les TICE à I'IUT1 de Grenoble

P. Huard

Institut Universitaire de Technologie 1 de Grenoble

Domaine universitaire

F-38402 Saint Martin d'Hères, France 


\title{
EXPERIENCE DE DEVELOPPEMENT ET DE MISE EN PLACE DE MODULES DE FORMATION PAR LES TICE A L'IUT1 DE GRENOBLE
}

\author{
Pierre HUARD \\ Institut Universitaire de Technologie 1 de Grenoble \\ Domaine Universitaire - 38402 Saint Martin d'Hères Cedex \\ Pierre.Huard@ujf-grenoble.fr \\ http://www-iut.ujf-grenoble.fr/DEV
}

\begin{abstract}
Résumé-La préparation d'un D.U.T., ou d'une licence professionnelle, se caractérise par l'acquisition de connaissances dans plusieurs champs disciplinaires, organisés en modules de formation. L'essor actuel des Technologies de l'Information et de la Communication, offre la possibilité de diffuser via le réseau Internet, une partie des éléments de formation d'un module. Nous présenterons tout d'abord la structure d'un module «TICE » qui permet à chaque enseignant de s'insérer dans le processus de formation, en lui donnant la possibilité d'exploiter son expérience et ses investissements antérieurs dans la conception de supports de formation. Nous présenterons ensuite les outils utilisés, et la stratégie mise en place pour permettre aux enseignants de développer et d'actualiser eux mêmes leur module « TICE ».
\end{abstract}

Mots clés-TICE, Enseignement à distance, Nouvelles approches pédagogiques, Multimédia.

\section{INTRODUCTION}

Durant la période 1998-2002, l'IUT1 de Grenoble à mis en place une organisation et une infrastructure permettant d'adapter les supports de formation utilisés par les enseignants des trois départements Génie Civil (GC), Génie Electrique (GEII) et Génie Mécanique (GMP) pour les rendre utilisables dans le cadre d'une préparation des formations à distance.

Cette préparation consiste à proposer aux étudiants de recevoir une partie de la formation à distance (l'ensemble des cours et des TD - travaux dirigés - de la formation en «présentiel») et une partie durant des séances de regroupement (l'ensemble des TP- travaux pratiques - de la formation en « présentiel »).

\section{STRUCTURE D’UN « MODULE TICE »}

\section{A. Avant propos}

La préparation d'un D.U.T. se caractérise par l'acquisition de connaissances dans plusieurs champs disciplinaires, organisés en modules de formation. En formation en "présentiel», chaque module est structuré de façon à atteindre des objectifs pédagogiques bien précis :

1. le Cours, permet de présenter aux étudiants les connaissances théoriques sur lesquelles repose un champ disciplinaire.

2. les séances de Travaux Dirigés (TD) permettent de mettre en place des méthodologies d'utilisation de ces connaissances à travers la résolution d'exercices et de vérifier ainsi la compréhension des concepts présentés dans le cours.
3. les séances de Travaux Pratiques (TP) permettent d'exploiter les connaissances acquises sur des situations et avec des matériels rencontrés dans un environnement industriel.

\section{B. Formation à distance}

L'essor actuel des Technologies de l'Information et de la communication, permet d'envisager la diffusion via le réseau Internet, d'une partie des éléments de formation d'un module. Les objectifs particuliers affectés aux Travaux Pratiques dans l'apprentissage cognitif, ne permettent pas d'envisager leur réalisation en dehors de séances effectuées en «présentiel». La composition d'un module de formation proposé dans le cadre d'un enseignement à distance est donc la suivante :

1. des "modules TICE", permettant de se substituer aux séances de Cours et de TD de la formation en « présentiel».

2. des Travaux Pratiques effectués en «présentiel » durant des séances de regroupement.

\section{Structure d'un module TICE}

De façon à permettre à chaque enseignant de s'insérer dans le processus de formation, en lui permettant d'exploiter son expérience et ses investissements antérieurs dans la conception de supports de formation, la structure proposée pour un « module TICE » est la suivante :

Un module est constitué d'un ensemble de chapitres.

Exemple: L'écran d'accueil d'un module présente l'ensemble des chapitres constituant le module. 


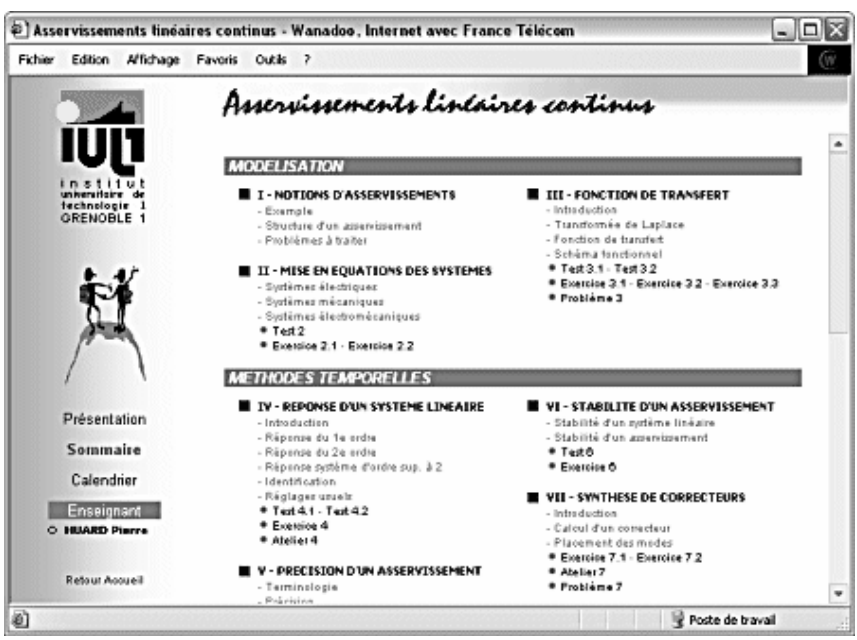

Un «Calendrier » planifie séance par séance le rythme de travail de l'étudiant.

Exemple: Calendrier d'étude du module «Asservissements linéaires continus».

Le calendnier subrant planife francemern wotre travail sur ce module. Le temps a consacrer á une seance est denviron $25 \mathrm{~h}$

o Calendrier.

\begin{tabular}{|c|c|c|c|c|c|}
\hline Chapities & Siancese & Lactuses & Tertt & Exerzicesthateliers & 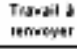 \\
\hline Chasite 1 & \multirow{2}{*}{ Siante 1} & 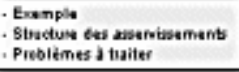 & & & \\
\hline \multirow[t]{2}{*}{ Chaspitse 2} & & 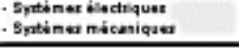 & - Teat 2 & - Exatesice 2.1 & \\
\hline & $\operatorname{sianse} 2$ & - Sptiner isectumbicaniquer & & - Extusiot 2.2 & \\
\hline \multirow[b]{2}{*}{ Chappites 3} & Seanes 3 & $\begin{array}{l}\text { - Intreduation } \\
\text { Trandoince de Laplace } \\
\text { Fornoson de trangren }\end{array}$ & - Tet 3.1. & - Exerese 3.1 & \\
\hline & Stante 4 & . Betiema fendianteal & - Tet 32 & 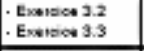 & \\
\hline
\end{tabular}

Chaque chapitre comporte un ensemble de «supports de formation » et un plan d'étude, précisant à l'étudiant le temps à consacrer à l'étude de ce chapitre.

Exemple: La page "Avant propos 》 précise les objectifs de ce chapitre ainsi que l'ordre dans lequel doit être exécutée chaque activité proposée.

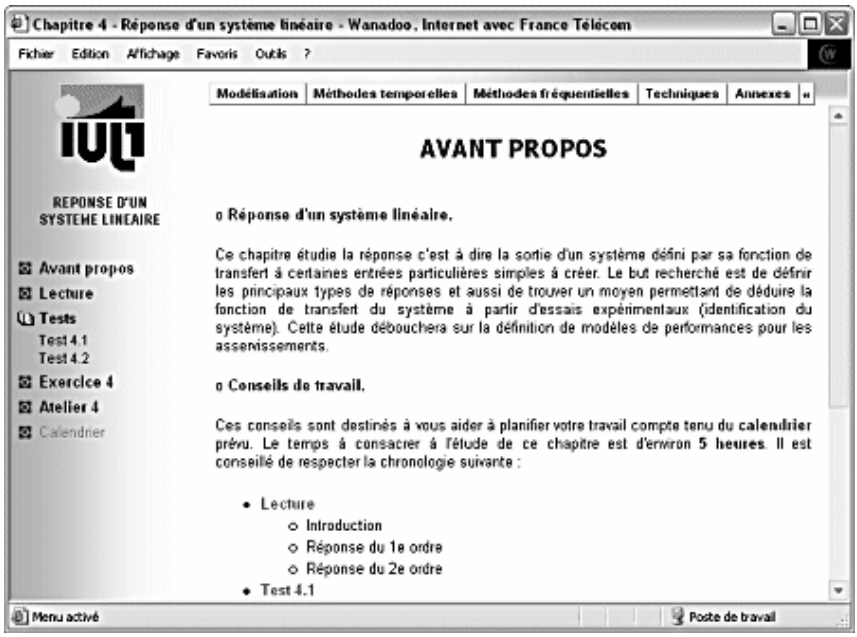

1. une partie cours («Lecture ») présente les principaux concepts à maîtriser. Cette partie diffusée aux étudiants au format «html» (ou pdf) peut être enrichie par des animations disponibles sur le réseau Internet et mises dans le domaine public par leurs auteurs.

2. un Q.C.M. («Test»), permet aux étudiants de s'autoévaluer sur les concepts présentés précédemment. Ce Q.C.M. a essentiellement pour but d'inciter l'étudiant à consulter le cours.

Exemple: L'étudiant peut à tout moment calculer son "Score ». Les numéros des questions correspondant à des réponses inexactes sont indiqués dans la zone de texte située dans la partie gauche de l'écran. Un chronomètre fixe le temps à consacrer à ce test.Le nombre de fois où l'étudiant peut calculer son "score" est paramétrable. Lorsque le temps limite est atteint ou lorsque l'étudiant a effectué le nombre maximum de calculs autorisés, un écran lui propose de consulter la solution ou de poursuivre.

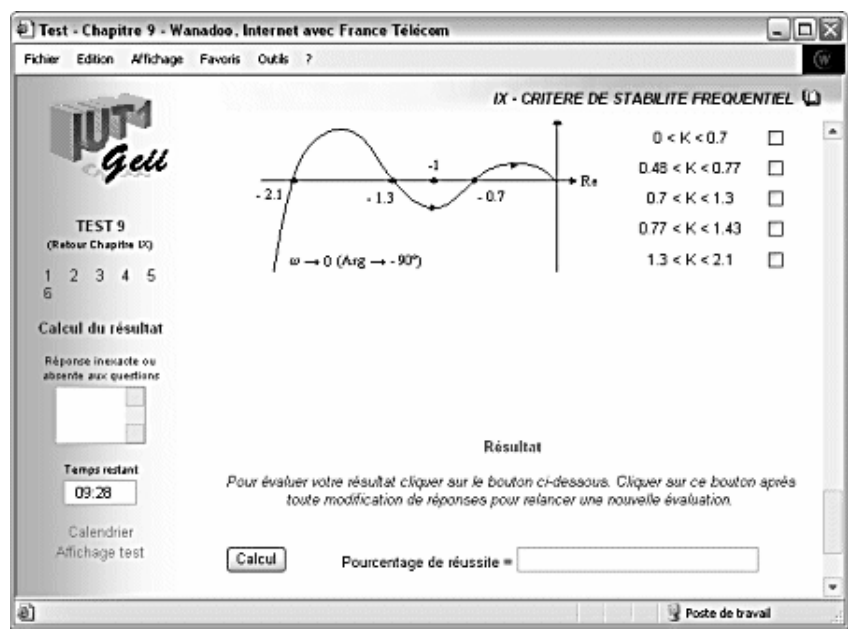

3. des exercices

- un exercice sans solution («Problème ») : un énoncé d'exercice est proposé à l'étudiant. L'étudiant renvoie son travail à l'enseignant pour correction et évaluation.

- un exercice avec solution : un énoncé d'exercice est proposé à l'étudiant. L'étudiant peut à tout moment consulter la solution de certaines questions. Cette solution est présentée sous la forme de différents tableaux qui, par leur enchaînement, permettent de reproduire la méthodologie utilisée par l'enseignant durant les séances de T.D. effectuées dans une formation en «présentiel ». L'accès à la solution des questions peut être protégé par un code d'accès qui est dévoilé aux étudiants durant des séances de «Travaux Dirigés virtuels » organisées autour d'un forum de discussion.

Exemple: Un énoncé d'exercice à résoudre est proposé à l'étudiant. L'étudiant travaille sur cet exercice de façon classique, sans avoir besoin de l'ordinateur. Dans cet exemple l'accès à la solution de la question 1 est autorisé ; l'accès aux solutions des questions 2 et 3 est protégé par un code d'accès. 


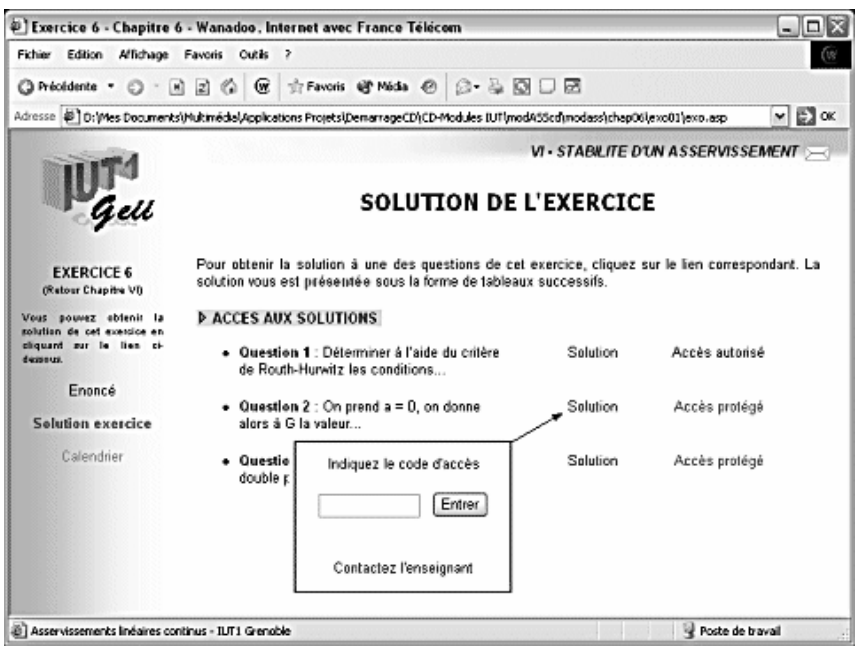

La solution est présentée sous la forme d'une animation HotMédia [1] sonorisée (un commentaire sonore accompagne l'enchaînement des différents tableaux présentant la solution).

- éventuellement un «Atelier» consistant en un exercice exploitant une ressource de «simulation » disponible sur le réseau Internet.

Exemple: Cet "Atelier» propose un exercice basé sur l'utilisation d'une applet (simulateur) mise en ligne par le "Korea Information Center for Chemical Engineering" (Corée).

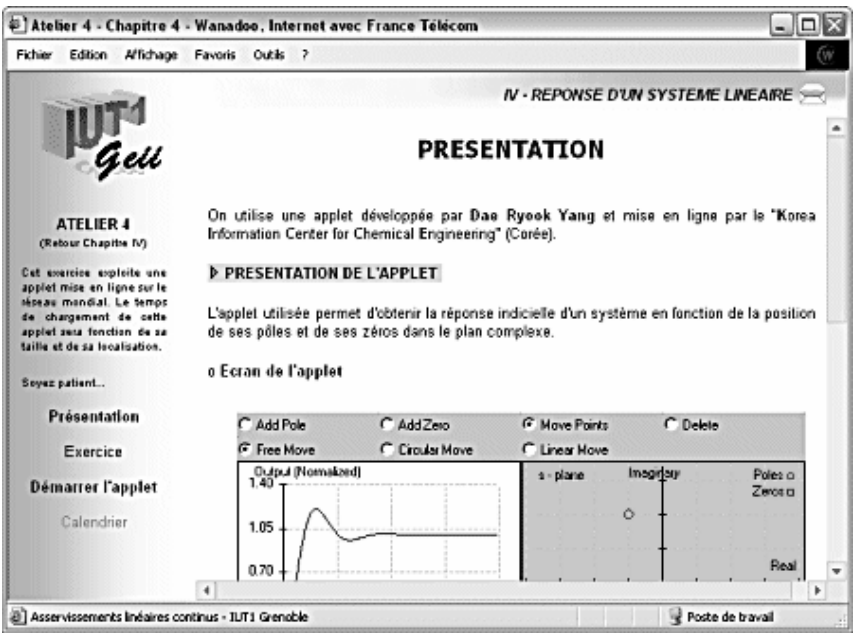

Enfin, une rubrique «Pour en savoir plus», permet à l'enseignant de présenter à l'étudiant une bibliographie et une webographie pertinentes relatives à ce module.

\section{ChOIX TECHNOLOGIQUES - DEVELOPPEMENT ET MISE EN PLACE DES MODULES}

\section{A. Choix technologiques}

Le module présenté précédemment a été réalisé à partir d'un modèle générique développé en interne à l'IUT. Ce modèle, indépendant de l'utilisation d'une plate-forme de e-learning, exploite uniquement les possibilités des langages html et javascript (version ECMA script interprété sur le poste client).

Cette solution permet :
1. un développement des modules à partir d'un simple éditeur html, d'un logiciel de dessin et d'un intégrateur de ressources multimédia (logiciel HotMédia [1]).

2. une installation des modules sur n'importe quel serveur Web ou une diffusion sur CD-Rom. Les solutions d'exercices (conçues dans HotMédia) sont lues dans le navigateur client par une applet Java.

La mise en place du dispositif d'enseignement à distance a nécessité, de la part du service informatique de l'IUT, l'installation et l'administration des éléments suivants sur un serveur dédié aux TICE :

1. un serveur Web

3. un serveur FTP

4. un forum de discussion

5. un portail d'accès aux modules (http://iut-tice.ujfgrenoble.fr)

\section{B. Développement et mise en place des modules}

Le développement des modules par les enseignants n'était envisageable qu'à condition que les enseignants s'approprient le «modèle générique» présenté précédemment. Chaque année, des séances de formation à l'utilisation et au paramétrage du «modèle générique » sont organisées à l'IUT.

Pour initier le développement des modules par les enseignants, nous avons bénéficié d'une aide financière de la part de GRECO [2] (Structure mise en place par les 6 établissements de l'enseignement supérieur de l'Académie de Grenoble). Au fur et à mesure du développement des modules, nous avons été amenés, en réponse aux interrogations des enseignants, à préciser plusieurs procédures relatives à l'édition et à l'utilisation des modules installés sur le serveur :

1. Validation des contenus : Placée sous la responsabilité du chef de département, les modules proposés par les enseignants sont validés par l'équipe pédagogique du département avant publication sur le serveur Web de l'IUT. L'enseignant auteur s'engage à intégrer dans son module un minimum de supports de formation permettant un travail en autonomie de l'étudiant. (éléments de cours, QCM, exercices avec solutions, plan d'étude du module,...)

2. Propriété intellectuelle et droit d'auteur : Nous avons été amenés à définir une "Charte de l'enseignement à distance de l'IUT1 » précisant :

- la limitation de l'accès aux modules publiés aux publics déterminés par la direction de l'IUT.

- la nécessité pour l'établissement d'obtenir de l'enseignant auteur une autorisation de publication des supports de formation créés; l'ensemble des modules étant rassemblés et organisés sous la direction éditoriale, pédagogique, administrative et technique de l'IUT1, pour constituer une œuvre collective au sens où l'entend le code de la propriété intellectuelle.

- l'obligation pour l'enseignant auteur d'obtenir l'accord écrit des responsables de sites et/ou des auteurs d'applets dans le cas de la mise en place de liens à partir du module en ligne vers d'autres sites et, a fortiori dans le cas d'utilisation d'applets mises en ligne sur d'autres sites. 
3. Suivi pédagogique du travail de l'étudiant: Un cahier des charges de l'enseignant «tuteur» précise le rôle et le travail à effectuer par l'enseignant dans le cadre du suivi à distance des étudiants inscrits dans un des trois cursus proposés à distance. Ce cahier des charges prévoit pour l'enseignant tuteur :

- la réponse aux questions des étudiants par échange de méls ;

- la correction du travail rendu et la communication aux étudiants du bilan de leur travail ;

- l'actualisation, chaque année, des supports de formation utilisés ;

- l'organisation, à la demande des étudiants, de plages de contacts synchrones ;

- la rédaction d'énoncés, la correction et la notation des copies des contrôles de connaissances prévus durant les périodes de regroupement ;

\section{BILAN ET PERSPECTIVES}

Durant la période 1998-2002, la réalisation de cette expérience s'est déroulée de la façon suivante :

- développement et mise au point du « modèle générique» de « module TICE ».

- mise en place d'un serveur d'information destiné aux enseignants impliqués dans cette action (http://wwwiut.ujf-grenoble.fr/DEV)

- découpage des modules de formation adaptés à la formation à distance. Ce travail a permis de définir les modules à développer et à numériser ainsi que les séances de regroupement à prévoir pour effectuer les TP et les contrôles de connaissances. En s'appuyant sur les programmes définis par les CPN des spécialités Génie Electrique, Génie Mécanique et Productique et Génie Civil et sur les travaux précédemment effectués sur le découpage en ECTS, il résulte que pour un DUT, environ 800 heures de formation seront dispensées à distance, le reste de la formation étant effectué durant des séances de regroupements consacrées aux travaux pratiques et au contrôle de connaissances.

- Organisation de sessions de formation pour les enseignants à l'utilisation du « modèle générique» de « module TICE ».

- mise en place d'un laboratoire pédagogique (géré par le service informatique de l'IUT) destiné aux enseignants pour développer leur module

- mise en place du serveur Web pédagogique par le service informatique de l'IUT. L'administration de ce serveur est sous la responsabilité du chef du service informatique de l'IUT. Chaque enseignant auteur dispose d'un accès FTP sur sa ressource et met à jour lui-même ses supports de formation.

\section{Perspectives}

L'IUT 1 de Grenoble se fixe comme objectif de continuer le développement de l'ensemble des modules nécessaires au bon fonctionnement de ce type de formation, ce qui implique :

- la formation de nouveaux enseignants à l'utilisation du «modèle générique » (actuellement 52 enseignants ont suivi les sessions de formation organisées par l'IUT)

- l'étude de la mise en place progressive d'outils de communication synchrone (chat, visioconférence...) chargé de compléter les supports pédagogiques développés par les enseignants.

- l'obtention de moyens permettant de continuer la politique d'incitation des enseignants à adapter leur instrument de formation. (environs 600 heures de formation sont actuellement disponible en ligne).

- l'étude de la mise en place éventuelle de ce type de formation dans d'autres spécialités (GTR).

Pour conclure mon exposé sur cette expérience, je citerai quelques unes des conditions «nécessaires et non suffisantes ", à la généralisation de l'usage des TICE dans l'enseignement supérieur, formulées dans une étude effectuée par la société ALGORA pour le Ministère de l'Education Nationale [3] :

1. rapprocher les deux catégories enseignants et IATOS, en associant les seconds aux projets pédagogiques, afin de mettre à profit leurs expertises et compétences ;

2. prendre en compte, dans les grilles d'avancement des enseignants, le développement, l'innovation pédagogique et la recherche sur l'amélioration des processus éducatifs ;

3. démontrer, par une projection et une anticipation sur plusieurs années, que le temps passé à chercher de nouveaux outils, à les développer ou à les faire utiliser, n'est pas un investissement à fond perdu, ni pour l'établissement, ni pour les enseignants ;

4. mettre en œuvre des modalités rationnelles de décompte des heures de travail, qui distinguent, de manière univoque, les différentes activités liées à l'enseignement, à l'amont, au suivi, à l'ingénierie ;

5. veiller à ce que l'infrastructure se mette au service des pratiques pédagogiques innovantes, en mettant à disposition des enseignants les moyens techniques et humains nécessaires.

\section{REFERENCES}

[1] HotMédia : Logiciel d'intégration de ressources multimédia développé par la société IBM. (http://www3.ibm.com/software/ad/hotmedia/)

[2] GRECO : GREnoble Universités Campus Ouvert. Structure mise en place par les 6 établissements de l'enseignement supérieur public de l'Académie de Grenoble, dans le but d'adopter une stratégie commune et cohérente, de déploiement des technologies d'information et de communication dans le domaine de l'éducation. (http://greco.grenet.fr/index.htm)

[3] «Analyse des besoins de formation des personnels de l'enseignement supérieur à l'usage des TICE dans le processus enseigner-apprendre » : Etude commanditée par le Ministère de l'Education Nationale, confiée à la société ALGORA et réalisée par

F.HAEUW (mars 2002)

(http://ressources.algora.org/reperes/competences/organi sme/besoins.asp) 